\title{
The rise of BioSAXS at the ESRF: BM29 Beamline for SAXS on Proteins in Solution
}

\author{
Petra Pernot ${ }^{(1)}$, Martha Brennich ${ }^{(2)}$ and Mark Tully ${ }^{(1)}$ \\ (1) ESRF-The European Synchrotron, 71 avenue des Martyrs, Grenoble, France \\ (2) EMBL Grenoble Outstation, 71 avenue des Martyrs, Grenoble, France
}

The first dedicated BioSAXS endstation at the ESRF, the undulator-based beamline ID14-3, entered user operation in 2008. In 2012, as part of Phase I of the ESRF upgrade program, ID14-3 was replaced by BM29, a new bending magnet-based BioSAXS station opened to users. Since 2008, the number of BioSAXS publications based on ESRF-collected data has grown near-exponentially and BM29 is now significantly oversubscribed. This is a worldwide trend as in recent years a combination of advances in sample handling, computer modelling and synchrotron sources have made BioSAXS more and more attractive. Moreover, whereas in the past even experienced crystallographers complained that SAXS experiments required too much time and effort, now they consider them indispensable.

BioSAXS experiments at BM29 are highly automated, offering two main modes of operation: BioSAXS with a robotic liquid handling sample changer (SC) and BioSAXS with online size-exclusion chromatography (SEC), purifying samples immediately prior to X-ray exposure. Switching between SC and SEC-SAXS modes is fully automated, maximizing the efficiency of the beamline. In SC operation up to a thousand measurements can be performed per day, with help from dedicated beamline control, dataacquisition software, real-time data display and automatic data processing. In both modes data collection parameters and results are logged and stored in the modified ISPyB database.

After the completion of the ESRF-Extremely Brilliant Source project in 2020, BM29 will have a new source, a 2-pole wiggler, to replace the current bending magnet. This will provide a smaller, more intense X-ray beam at the sample position. This will be coupled with a new, in vacuum detector while the current BioSAXS sample environment will be modified accordingly. These upgrades will generate high quality, low noise data with lower concentrations of sample $(0.1 \mathrm{mg} / \mathrm{ml})$ necessary.

Here the experimental setups available on BM29 will be described together with the various examples of the BioSAXS data obtained. Recent examples of BM29 research, including the integration of microfluidics systems and online ion-exchange chromatography as well as the possibilities for new experiments in BioSAXS. 